Peter J. Strouse

D. Gregory Bates

David A. Bloom

Mitchell M. Goodsitt

\section{Non-contrast thin-section helical CT of urinary tract calculi in children}

Received: 1 May 2001

Accepted: 26 October 2001

Published online: 2 February 2002

(c) Springer-Verlag 2002
P.J. Strouse $(\square)$

Section of Pediatric Radiology,

C.S. Mott Children's Hospital,

University of Michigan Medical Center, 1500 East Medical Center Drive,

Ann Arbor, MI 48109-0252, USA

E-mail: pstrouse@umich.edu

Tel.: + 1-734-7632570

Fax: + 1-734-7649351

D.G. Bates

Department of Radiology, Columbus Children's Hospital, 700 Children's Drive, Columbus, OH 43205, USA

D.A. Bloom

Department of Pediatric Imaging, Children's Hospital of Michigan, 3901

Beaubien Blvd, Detroit, MI 48201, USA

M.M. Goodsitt

Section of Radiology Physics, Department of Radiology, University of Michigan

Medical Center, 1500 East Medical Center

Drive, Ann Arbor, MI 48109-0252, USA
Abstract Background: Non-contrast thin-section helical CT has gained acceptance for the diagnosis of urinary tract calculi in adults, but experience with the technique in children is limited. Purpose: To evaluate the utility of non-contrast thin section helical CT for the diagnosis of urinary tract calculi in children. Materials and methods:

Radiology databases at three pediatric institutions were searched to identify all pediatric patients evaluated by "renal stone" protocol CT scans (no oral or intravenous contrast, scans covering the entire urinary tract obtained in helical mode with narrow collimation ( $\leq 5 \mathrm{~mm})$ ). CT scans were reviewed for the primary finding of urinary tract calculi, for secondary signs of acute urinary tract obstruction and for evidence of alternative diagnoses. Medical records were reviewed to determine clinical presentation and to confirm the eventual diagnosis. Results: One hundred thirty-seven scans of 113 children (mean age: 11.2 years) were studied. Thirty-eight of 94 examina- tions $(40 \%)$ performed on 82 children for acute pain and/or hematuria showed ureteral calculi. Alternative diagnoses were suggested by CT on 16 scans $(17 \%)$. Twenty-eight scans were performed on 10 asymptomatic children with known calculus disease confirming renal stone burden on 21 scans $(75 \%)$ and persistent ureteral calculi on 6 scans $(21 \%)$. Upper tract calculi were demonstrated on 10 of 15 scans $(67 \%)$ performed to evaluate for calculi in patients with known non-calculus genitourinary tract abnormalities. Conclusions: Non-contrast thin section helical CT is a useful method to diagnose urinary tract calculi in children. Radiation dose in this retrospective study may exceed the lowest possible radiation dose for diagnostic accuracy. Further research is needed to optimize $\mathrm{CT}$ imaging parameters, while maintaining diagnostic accuracy and minimizing radiation dose.

Keywords Thin-section helical CT · Urinary tract calculi

\section{Introduction}

The concept of using non-contrast helical computed tomography (CT) to evaluate for urinary tract calculi was introduced in 1995 by Smith et al. [1]. Since then, several investigators have researched this technique in wholly or predominantly adult patient populations.
Advantages of non-contrast thin section helical CT over excretory urography include: (1) shorter examination times; (2) higher sensitivity and specificity for calculi; (3) no need for intravenous contrast; (4) a greater potential for making alternative diagnoses [1, 2, 3]. Disadvantages include: (1) a higher radiation dose to the patient using most published protocols; and, (2) higher cost and 
therefore higher charge to the patient [4]. Application of this technique in the pediatric population has been limited. Urinary tract calculi account for between 1 per 1,000 and 1 per 7,600 pediatric hospital admissions [5]. The incidence of calculi in children is less than $2 \%$ of the reported incidence in adults [6]. Urinary tract calculi are thus a much less frequent problem in children than in adults; however, they do occur and often pose a challenge in diagnosis.

Our objective in this investigation was to examine the use of renal stone protocol CT in a pediatric population.

\section{Materials and methods}

The study was a multi-institutional, retrospective review. Institutional review board approval was obtained at two institutions and was waived for retrospective review at the third. Radiology databases at three pediatric hospitals were reviewed from 1997 to February 2000 to identify patients undergoing "renal stone" protocol CT for the evaluation of urinary tract calculi. Inclusion criteria were: (1) age $\leq 18$ years; (2) helical scanning mode; (3) $\leq$ $5 \mathrm{~mm}$ collimation; (4) no intravenous contrast; (5) scans covering the entire urinary tract.

Apart from the aforementioned criteria, CT parameters were not standardized. The majority of scans were performed with one of three protocols: (1) $5 \mathrm{~mm}$ collimation, 1:1 pitch, 180-280 mAs; (2) $5 \mathrm{~mm}$ collimation, 1.5:1 pitch, $120-240 \mathrm{mAs}$; (3) $5 \mathrm{~mm}$ collimation, 1:1 pitch, 180-280 mAs from above kidneys to iliac crests with $3 \mathrm{~mm}$ collimation, 2:1 pitch, 140-240 mAs from iliac crests to below the bladder. All scans were performed with $120 \mathrm{kVp}$. All but three scans were performed on GE Hi-Speed Advantage helical scanners (GE Medical Systems, Milwaukee, Wis.). Three patients were scanned on a GE Lightspeed multi-helical scanner (GE Medical Systems, Milwaukee, Wis.). All scans were performed with the patient supine. In a few instances, limited prone images were obtained to differentiate an impacted calculus at the ureterovesical junction from a bladder calculus.

Images from one institution were reviewed by a single observer (PJS) with consensus readings by two of the authors (PJS and DGB or PJS and DAB) at the other two institutions. Hard-copy images were reviewed for the presence, number and location of renal and ureteral calculi. Any round or oval opacity of mineral density projecting within the kidney or ureter was considered a calculus. Secondary findings of ureteral calculi were tabulated, including hydronephrosis, increased renal size (vs the contralateral kidney), decreased renal attenuation (vs the contralateral kidney), perinephric stranding, hydroureter, periureteric stranding, tissue rim sign and uterovesical junction edema $[7,8,9,10]$. Alternative diagnoses and other findings, unrelated to acute presentation, were recorded.

Medical records, when obtainable, were reviewed to determine patient presentation, and clinical and imaging follow-up, including final diagnosis. Records of private patients referred for imaging only were often unavailable (35 patients). For further analysis patients were divided into three groups based on the initial reason for imaging: (1) acute presentation with pain and/or hematuria; (2) asymptomatic, follow-up of known calculus disease; (3) known genitourinary tract anomaly or non-calculus disease. Multiple scans on the same patient were evaluated separately. If the patient was symptomatic at the time of scanning, the scan was included in the acute presentation group. Some patients in group 3 presented with acute symptoms, but were analyzed separately as the preexisting abnormality might affect the CT findings, particularly in reference to secondary signs of acute ureteral obstruction. For patients in the acute presentation group the incidence of pain and hematuria was tabulated. Pain was recorded as "flank pain" or "other pain." If the site was not specified the pain was recorded as "other pain." Hematuria was considered present if either gross or microscopic. If pain or hematuria was not recorded as present, it was considered to be absent. Some patients in group 2 had microscopic hematuria, but were otherwise asymptomatic.

Scans with ureteral calculi were considered true positive for ureteral calculus if subsequent clinical records or imaging studies (excretory urography, CT with contrast, retrograde ureterogram) were consistent with or confirmed the diagnosis. Scans with CT demonstrated ureteral calculi in patients whose symptoms resolved with hydration and/or in whom calculi were physically recovered were considered true positive. Scans with ureteral calculi were considered false positive if subsequent clinical records or imaging studies indicated an alternative cause for the patient's symptoms. Scans without ureteral calculi were considered true negative if subsequent clinical records or imaging studies identified an alternative diagnosis. Scans without ureteral calculi were considered false negative if there was subsequent clinical or imaging confirmation of ureteral calculi. Scans were considered indeterminate if there was inadequate clinical and imaging follow-up to confirm the presence or absence of a calculus.

\section{Results}

A total of 163 non-contrast helical CT scans performed to evaluate urinary tract calculi were identified. Twentysix scans were excluded for reasons of age greater than 18 years (10), collimation greater than $5 \mathrm{~mm} \mathrm{(8),} \mathrm{ab-}$ dominal imaging only (8), scans unavailable for review (2), and pelvic imaging only (1). One hundred thirtyseven scans of 113 patients met the inclusion criteria. The ages ranged from 1 to 18 years with a mean of 11.2 years. There were 52 females and 61 males studied.

Ninety-four scans of 82 patients were performed for an acute presentation of pain and/or hematuria (group 1). Twenty-eight scans of 10 patients were performed on asymptomatic patients to follow-up known stone disease (group 2). Fifteen scans of 14 patients were performed on patients with a known genitourinary tract abnormality to assess for calculi (group 3).

Table 1 lists the frequency of renal and ureteral calculi identified in group 1 patients based on the presenting symptoms. Fifty percent of patients with flank pain and hematuria were found to have a ureteral calculus. In the absence of both flank pain and hematuria, the incidence of ureteral calculus was 17\% (3 of 18 patients). All of these patients presented with abdominal pain, but not specifically flank pain. Forty-three ureteral calculi were identified in 41 ureters of 38 patients. Ureteral calculi were identified at the ureteropelvic junction (4), proximal ureter (5), mid-ureter (2), distal ureter (9), and ureterovesicular junction (23). Two patients had single bilateral ureteral calculi and one patient had one right and three left ureteral calculi. Seventeen of 38 patients with ureteral calculi also had renal calculi. Four patients without ureteral caluculi had renal calculi. Secondary signs of ureteral calculi and acute urinary tract 
Table 1 Presenting symptoms and urinary tract calculi in patients with acute pain and/or hematuria (group 1)

\begin{tabular}{lcccc}
\hline Symptoms & Number & $\begin{array}{l}\text { Ureteral calculi } \\
\text { only }\end{array}$ & $\begin{array}{l}\text { Ureteral and } \\
\text { renal calculi }\end{array}$ & $\begin{array}{l}\text { Renal calculi } \\
\text { only }\end{array}$ \\
\hline Flank pain and hematuria & 42 & 10 & 11 & 0 \\
Flank pain & 12 & 3 & 3 & 1 \\
Hematuria & 6 & 1 & 1 & 0 \\
Other pain and hematuria & 16 & 5 & 1 & 1 \\
Other pain & 18 & 2 & 1 & 2 \\
Total & 94 & 21 & 17 & 4 \\
\hline
\end{tabular}

obstruction for patients in group 1 are included in Table 2 and illustrated in Figs. 1 and 2. An overwhelming majority $(93 \%)$ of patients with ureteral calculi showed at least one secondary sign. Single phleboliths were identified in two patients and were easily distinguishable from urinary tract calculi based on their anatomical location. One patient with polyarteritis nodosa had multiple vascular calcifications in the pelvis.

Of the 38 scans for acute presentation and ureteral calculi on CT, $30(79 \%)$ were true positive and eight $(21 \%)$ indeterminate. Of 56 scans for acute presentation and no ureteral calculi on CT, $24(43 \%)$ were true negative and $32(57 \%)$ indeterminate. The large number of indeterminate negative scans was due to two factors lack of an alternative diagnosis on follow-up (our definition of true negative required an alternative diagnosis be made on follow-up) or lack of access to clinical follow-up. There were no proven false-positive or falsenegative scans. Alternative diagnoses were identified by CT in $16(29 \%)$ of the 56 negative scans: cystitis (3), constipation (2), probable mesenteric adenitis (2), bilateral pyelonephritis (1), unilateral pyelonephritis (1), ureteropelvic junction obstruction (1), large bladder calculus (1), malrotation with volvulus (1), intussusception (1), typhlitis (1), peritonitis (1), and adnexal cyst (1) (Fig. 3). Nine patients had significant incidental findings, unrelated to the presenting symptoms: appendicolith (3), atrophic kidney (2), gallstone (1), colonic foreign body (1), bilateral L5 spondylolysis (1), and ovarian cyst (1).

Of the 42 patients in group 1 with presenting symptoms of flank pain and hematuria the final clinical diagnoses were: ureteral calculus $(21,11$ of whom also had renal calculi), urinary tract infection (4 - pyelonephritis (2); cystitis with possible pyelonephritis (2)), passed calculus prior to CT (1), ureteropelvic junction obstruction (1), post-streptococcal glomerulonephritis (1), IgA nephropathy (1), bacteremia of unknown etiology (1), constipation (1), ovarian cyst rupture (1), musculoskeletal pain (1), and no diagnosis (9).

Findings on the 28 scans in group 2 were: ureteral stones $(6,1$ bilateral), renal stones $(21,13$ bilateral), ureteral stents (11, 1 bilateral), ureteral stent and stone in the same ureter (1), encrusted stent (2, same patient). The presence of a ureteral stent required review and filming of images at bone window settings to eliminate
Table 2 Secondary signs of ureteral calculi in symptomatic patients (group 1)

\begin{tabular}{lcc}
\hline Secondary sign & $\begin{array}{l}\text { Side with } \\
\text { calculus }^{\mathrm{a}}\end{array}$ & $\begin{array}{l}\text { Side without } \\
\text { calculus }\end{array}$ \\
\hline Hydronephrosis & $30(73 \%)$ & $5(3 \%)$ \\
Hydroureter & $27(66 \%)$ & $2(1 \%)$ \\
Increased renal size & $26(63 \%)$ & $7(5 \%)$ \\
Perinephric stranding & $17(41 \%)$ & $3(2 \%)$ \\
Tissue rim sign & $14(34 \%)$ & 0 \\
Periureteric stranding & $13(32 \%)$ & 0 \\
Decreased renal attenuation & $10(24 \%)$ & 0 \\
Ureterovesical junction edema & $3(7 \%)$ & 0 \\
No secondary signs & $3(7 \%)$ & $141(96 \%)$ \\
Total & 41 & 147 \\
\hline
\end{tabular}

${ }^{\text {a }}$ Percentages in parentheses indicate percent with calculus (first column) or without calculus (second column) with the sign

streak and beam hardening artifact from the stent (Fig. 4).

Findings on the 15 scans in group 3 were: ureteral calculi (1, bilateral), renal calculi $(9,6$ bilateral), bladder calculi (4), atrophic kidney (2), upper pole renal scar (1), probable ureteropelvic junction obstruction (1). Underlying genitourinary anomalies or disease included: neurogenic bladder (4), prior repair of cloacal exstrophy (4), urinary tract infections (3, 1 with known vesicoureteral reflux), prior pyeloplasty for ureteopelvic junction obstruction (1), horseshoe kidney with recurrent urinary tract infection (1), cystitis cystica (1), prior renal trauma with surgery (1).

\section{Discussion}

The initial evaluation of children with an acute presentation of suspected urinary tract calculi is a complete history and physical examination. If the clinical presentation is classic, particularly in a patient with known stone disease or a predisposition to stone disease, then further imaging may not be necessary. Such patients can be managed with analgesics and hydration. Should symptoms fail to abate or if the presentation is not diagnostic for an impacted ureteral calculus, then imaging might be sought. While it might be argued that all patients with flank pain and hematuria be given a trial of pain medicine and hydration, it is clear that not every 

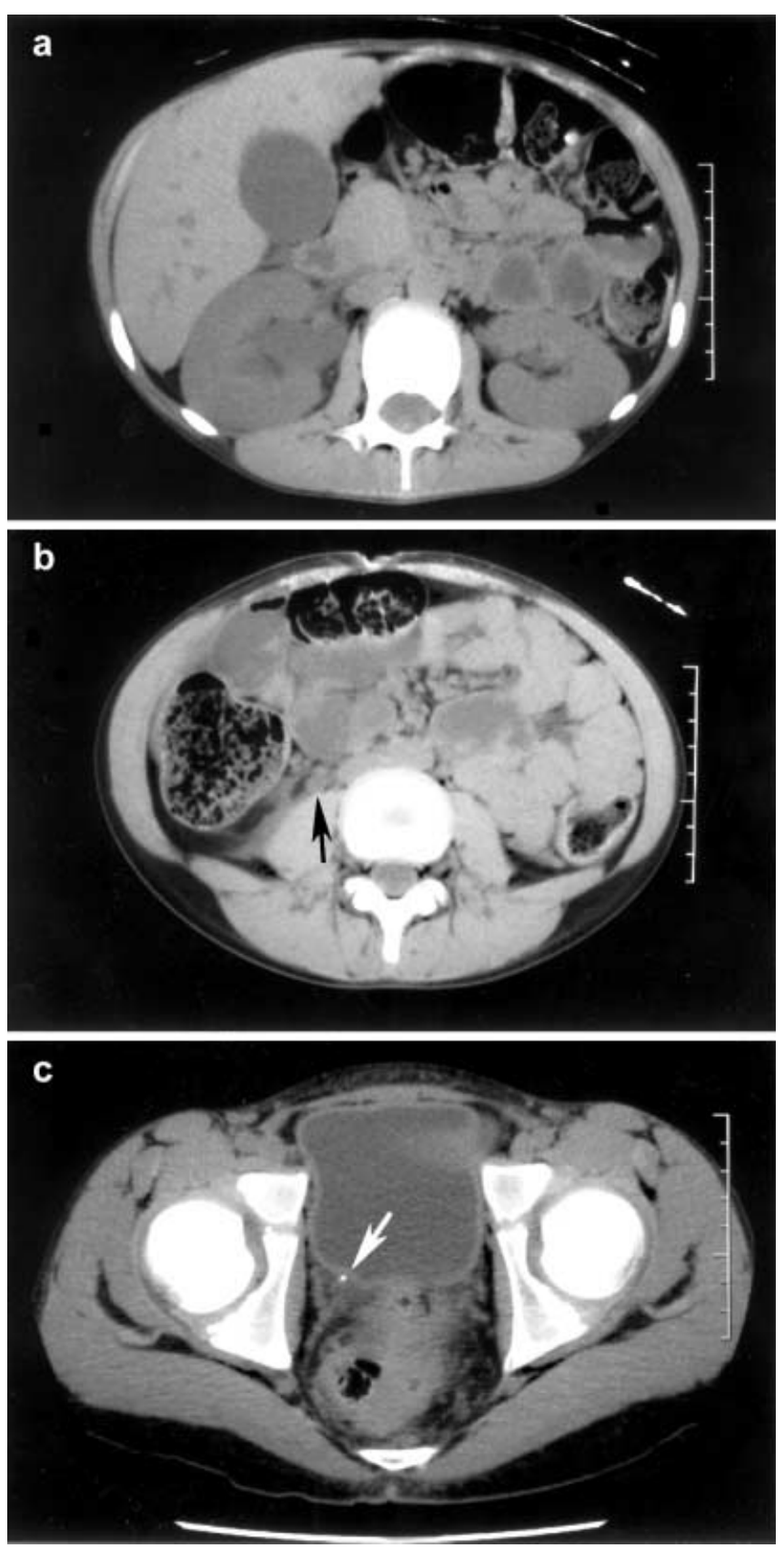

Fig. 1 An 11-year-old boy with right flank pain and hematuria. a CT scan at level of the kidneys shows mild right hydronephrosis and renal enlargement. b CT scan in lower abdomen shows dilatation of the right ureter (arrow) and periureteric stranding. c CT scan in the pelvis shows a calculus (arrow) impacted at the right ureterovesical junction with mild adjacent bladder wall thickening. This image was obtained with lower $\mathrm{mAs}$, narrower collimation and greater pitch than the higher images, accounting for a slight difference in image quality

child with flank pain and hematuria has a ureteral calculus. Some information is thus gained by imaging these patients.

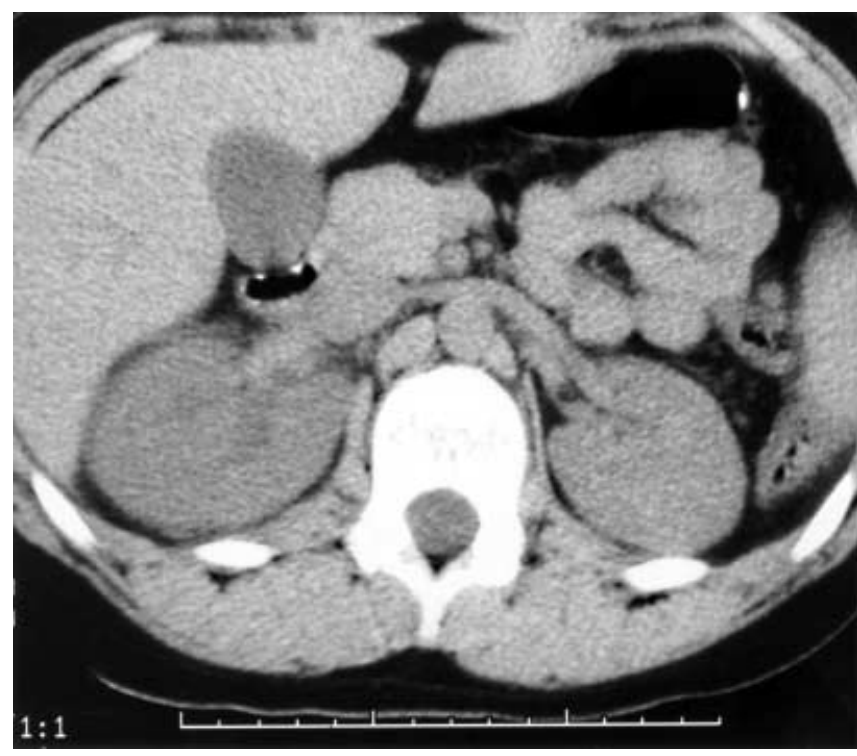

Fig. 2 A 12-year-old boy with a distal right ureteral calclus. CT scan at the level of the kidneys shows mild enlargement and subtle decrease in attenuation of the right kidney. Margins of the right kidney are indistinct due to perinephric stranding. The collecting system is not dilated

Although sonography offers the distinct advantage of no ionizing radiation, its ability to detect acute urinary tract obstruction and ureteral calculi is limited [11]. Sonography can confirm renal calculi, may show mild collecting system dilatation due to obstruction, and occasionally demonstrates a distal ureteral or ureterovesical junction calculus. Traditionally, excretory urography has been used to assess for ureteral calculi. Although excretory urography provides functional assessment of the degree of obstruction, definite disadvantages of the examination exist, most notably a potentially long examination time and the need for intravenous contrast administration.

CT provides the advantage of a short examination time. Patients can be scanned emergently and expediently in unscheduled time slots between scheduled patients. As no intravenous contrast is administered, no preparation is required and the attendant risks and costs of iodinated contrast are avoided. Studies in adults have shown that thin section helical CT has a greater sensitivity and specificity for urinary tract calculi than excretory urography $[1,2,3]$. Although the functional assessment of the degree of obstruction is lost, the detection of secondary signs of urinary tract obstruction on CT provides evidence as to the significance of an identified ureteral calculus. As shown in our series, CT has the potential to make alternative diagnoses. Although this appears to be an advantage, at least one study in adults has demonstrated a declining positive prediction rate and increasing rate of alternative diagnoses over time, indicating a propensity of the clinical 


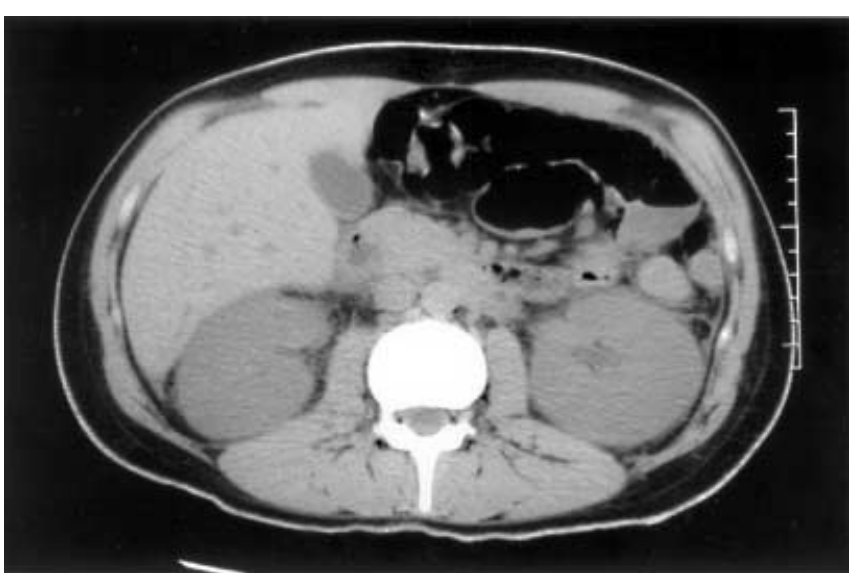

Fig. 3 An 18-year-old boy with an alternative diagnosis of bilateral pyelonephritis. The patient had both flank pain and hematuria. On CT both kidneys are enlarged and have indistinct margins; however, there was no collecting system or ureteral dilatation nor calculi. The diagnosis of pyelonephritis was suspected after this scan and subsquently confirmed by urinalysis and CT one day later with intravenous contrast

services to use the renal stone protocol as a "shot-gun" diagnosis tool [12].

Our clinical colleagues have also found the thinsection helical CT protocol helpful in following patients with known calculus disease. Although these patients are chiefly followed by clinical parameters and sonography, $\mathrm{CT}$ is occasionally valuable in mapping renal stone burden and determining if there is a persistent ureteral calculus. Thin-section helical CT is also occasionally helpful in evaluating the patient with a known abnormality of the genitourinary tract and suspected calculi. In these patients, body habitus or abnormal anatomy may render evaluation with sonography difficult, renal insufficiency may preclude contrast administration or render it suboptimal, and underlying urinary tract abnormalities may make the detection and interpretation of secondary signs of obstruction difficult. Both in patients with prior calculus disease and in those with other known genitourinary tract abnormalities, care must be taken in interpreting secondary signs which can be affected by underlying disease or prior intervention.

The incidence of hydronephrosis, hydroureter and increased renal size in our pediatric patients with ureteral calculi is less than, but approaches the incidence seen in adult series [7, 8, 9]. The incidence of perinephric stranding, periureteric stranding, the tissue rim sign and ureterovesical junction edema is substantially less than seen in adult series [7, 8, 9, 10]. Although these findings may be truly less common, the relative paucity of fat in the pediatric abdomen and pelvis renders identification of these findings more difficult. The interval between symptom onset and

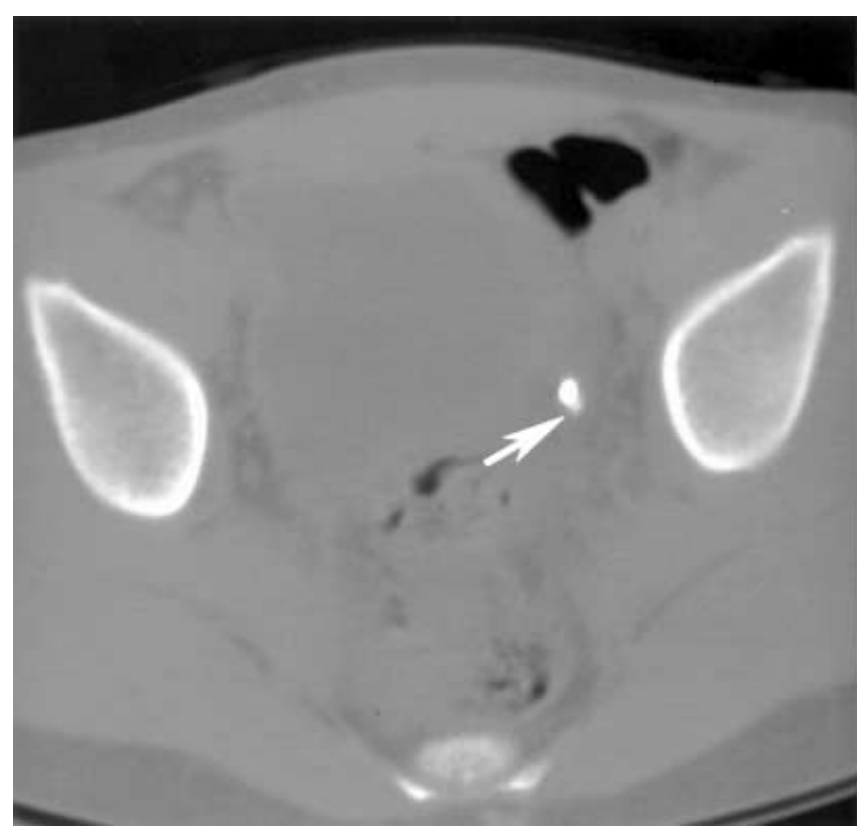

Fig. 4 A 6-year-old asymptomatic boy with known calculus disease and left ureteral stent. By manipulating CT window and level settings to near bone windows, a small calculus (arrow) is seen adjacent to the stent. The calculus was not visible on soft tissue windows. This calculus required removal via ureteroscopy

imaging may be shorter in children than adults, not allowing for secondary signs to develop. Phleboliths are rarely a diagnostic problem in children. For this reason, the tissue rim sign, which is used to distinguish phleboliths (no tissue rim) from ureteral calculi (with tissue rim), is of less utility. Secondary signs were infrequently seen in children without ureteral calculi. In some patients, the secondary signs were due to an alternative diagnosis such as pyelonephritis or ureteropelvic junction obstruction.

There were few patients with secondary signs suggesting the presence of an obstructing or partially obstructing calculus in whom no calculus was detected. We strongly suspect that some of our patients with indeterminate negative scans had passed a calculus prior to CT, although none of them showed the supportive secondary signs. It is unknown how rapidly the secondary signs resolve after a calculus is passed. Smaller calculi, which pass more readily, may produce fewer secondary signs especially if they do not lead to high-grade obstruction. Alternatively, our clinical colleagues and technologists may be effectively screening patients with passed calculi from proceeding to CT. It is also possible that some of our patients with indeterminate negative scans had small calculi missed by CT. The lack of secondary signs suggests this is not the case. None of these patients returned for further treatment, suggesting that if a calculus was missed, it was most likely clinically insignificant. 
The cost of a conventional CT scan of the abdomen, with or without contrast, is substantially higher than that of excretory urography. Accordingly, the charge to the patient is substantially higher, too. In order for thinsection helical CT to supersede excretory urography, the cost and patient charge differentials must be diminished. At two of the three institutions in this study, there is a substantially reduced charge for renal stone protocol CT compared to conventional CT. This charge is still on the order of twice that of excretory urography.

A limitation of this study is the variation in CT protocols between the three institutions. This is due to the retrospective approach of the study. In general, except for a few very young children, collimation was not varied based on age. Further study is needed to determine optimal collimation and pitch as a function of patient size. Another limitation is the lack of physical proof of calculus (calculus retrieval and laboratory analysis) in most patients. While it is possible that other densities were misidentified as calculi due to bias due to a relatively high pretest probability, misdiagnosis is unlikely given the high prevalence $(93 \%)$ of secondary signs. We did not attempt to assess interobserver or intraobserver variability in the identification of calculi or the assessment of secondary signs.

The radiation dose incurred with $\mathrm{CT}$ must be carefully considered before deciding to proceed with CT evaluation for urinary tract calculi [4]. CT is a major component of radiation exposure to children in diagnostic imaging. Table 3 lists the estimated radiation dose for excretory urography and each of the commonly used CT protocols in this study. The doses listed in Table 3 are calculated using several sources from the literature and making several assumptions $[13,14,15]$. It must be stressed that these are approximations. The doses also do not account for variations in the excretory urography radiation dose due to the use of different X-ray beam spectra ( $\mathrm{kVp}$ and beam filtration) and screen-film speed or variations in CT radiation dose associated with scanners of different models and makes due to differences in geometric magnification and X-ray beam spectra. Measurement of the actual dose incurred during helical CT is difficult. The radiation doses for CT in
Table 3 were calculated using $200 \mathrm{mAs}$ across the range of patient ages. From these calculations, it appears that for the adult the radiation dose for thin-section helical CT is nearly equivalent to that of a four-film excretory urogram. However, in younger children, maintaining the same mAs, the radiation dose appears to considerably higher with $\mathrm{CT}$. The chief modes of decreasing radiation dose in helical CT are increasing pitch and decreasing mAs [16]. Changing the pitch from 1:1 to $2: 1$ essentially halves the radiation dose. Dose is linearly related to mAs. Our empiric observations during review of scans with a range of mAs (120-280) are that scans at the lower end of the range were of near equal quality to those of higher mAs. Data in the literature supports the use of reduced $\mathrm{mAs}$ in pediatric CT scanning $[17,18,19]$. The last line of Table 3 provides dose estimates for CT using a pitch of $2: 1$ and an mAs of 100 . With these parameters, CT can be performed with less radiation dose than excretory urography in the older child and near equal doses at the mean age of patients in our study. It is unproven, however, whether these parameters allow for accurate detection of calculi. These radiation dose approximations do not account for higher effective radiation dose and greater stochastic radiation risks of carcinogenesis and genetic effects that exist in children [20].

In summary, we have found thin-section helical CT without intravenous contrast useful in diagnosing urinary tract calculi in children. We have found thin-section helical CT helpful in assessing for urinary tract calculi in children with (1) acute symptoms of ureteral calculus, (2) with known calculus disease for assessment of stone burden, and (3) with known genitourinary tract malformation and suspicion for calculi. CT offers distinct advantages over excretory urography; however, these advantages must be balanced against a higher cost and radiation dose incurred. If the cost and radiation dose of CT approach that of excretory urography, CT may be the preferred modality. Further research is necessary to optimize CT imaging parameters (pitch, collimation, mAs), while maintaining diagnostic accuracy and decreasing radiation dose to the lowest possible level.

Table 3 Estimated radiation dose (approximate organ dose to the ovary in Rads) ${ }^{\mathrm{a}}[13,14,15]$

\begin{tabular}{llllll}
\hline & Adult & 15 years old & 10 years old & 5 years old & 1 year old \\
\hline $\begin{array}{l}\text { Execretory urography } \\
\text { 4 films, 76 KVp }\end{array}$ & 0.40 & 0.23 & 0.17 & 0.11 & 0.07 \\
CT - 200 mAs, 120 KVp & $(32 \mathrm{mAs})$ & $(15 \mathrm{mAs})$ & $(6.4 \mathrm{mAs})$ & $(3.2 \mathrm{mAs})$ & $(2 \mathrm{mAs})$ \\
$5 \mathrm{~mm}$ collimation, 1:1 pitch & 0.54 & 0.61 & 0.77 & 0.90 & 1.07 \\
$5 \mathrm{~mm}$ collimation, 1.5:1 pitch & 0.36 & 0.40 & 0.51 & 0.60 & 0.71 \\
$3 \mathrm{~mm}$ collimation, 2:1 pitch & 0.27 & 0.31 & 0.39 & 0.45 & 0.54 \\
CT - 100 mAs, 120 KVp & 0.14 & 0.15 & 0.19 & 0.23 & 0.26 \\
5 mm collimation, 2:1 pitch & 0.19 & & \\
\hline
\end{tabular}

\footnotetext{
${ }^{\mathrm{a} A s s u m e s ~ o v a r y ~ t o ~ b e ~ a t ~ c e n t e r . ~ O v a r i a n ~ d o s e ~ f o r ~ C T ~ w i l l ~ b e ~ s l i g h t l y ~ h i g h e r ~ i f ~ p o s i t i o n e d ~ a w a y ~ f r o m ~ c e n t e r ~}$
} 


\section{References}

1. Smith RC, Rosenfield AT, Choe KA, et al. (1995) Acute flank pain: comparison of non-contrast-enhanced CT and intravenous urography. Radiology 194:789-794

2. Fielding JR, Steele G, Fox LA, et al (1997) Spiral computerized tomography in the evaluation of acute flank pain: a replacement for excretory urography. J Urol 157:2071-2073

3. Sommer FG, Jeffrey RB Jr, Rubin GD, et al. (1995) Detection of ureteral calculi in patients with suspected renal colic: value of reformatted noncontrast helical CT. AJR 165:509-513

4. Denton ERE, Mackenzie A, Greenwell T, et al. (1999) Unenhanced helical CT for renal colic - is the radiation dose justifiable? Clin Radiol 54:444-447

5. Santos-Victoriano M, Brouhard $\mathrm{BH}$, Cunningham RJ III (1998) Renal stone disease in children. Clin Pediatr 37:583600

6. Gill WB (1984) Renal calculus disease: classifications, demographics and etiological considerations. Semin Urol $2: 1-11$
7. Smith RC, Verga M, Dalrymple N, et al (1996) Acute ureteral obstruction: value of secondary signs on helical unenhanced CT. AJR 167:1109-1113

8. Katz DS, Lane MJ, Sommer FG (1996) Unenhanced helical CT of ureteral stones: incidence of associated urinary tract findings. AJR 166:1319-1322

9. Fielding JR, Fox LA, Heller H, et al. (1997) Spiral CT in the evaluation of flank pain: overall accuracy and feature analysis. JCAT 21:635-638

10. Heneghan JP, Dalrymple NC, Verga M, et al. (1997) Soft-tissue "rim" sign in the diagnosis of ureteral calculi with use of unenhanced helical CT. Radiology 202:709-711

11. Laing FC, Jeffrey RB, Wing VW (1985) Ultrasound versus excretory urography in evaluating acute flank pain. Radiology 154:613-616

12. Chen MYM, Zagoria RJ, Saunders HS, et al. (1999) Trends in the use of unenhanced helical CT for acute urinary colic. AJR 173:1447-1450

13. Huda W, Atherton JV, Ware DE, et al. (1997) An approach for the estimation of effective radiation dose at CT in pediatric patients. Radiology 203:417-422

14. Harrison RM (1981) Center axis depth-dose data for diagnostic radiology. Phys Med Biol 26:657-670
15. Foley WD, Jacobson DR, Begun FP (2000) Helical CT and renal calculi (reply to letter). AJR 174:568-569

16. Donnelly LF, Emery KH, Brody AS, et al. (2001) Minimizing radiation dose for pediatric body applications of single-detector CT: strategies at a large children's hospital. AJR 176:303-306

17. Paterson A, Frush DP, Donnelly LF (2001) Helical CT of the body: are settings adjusted for pediatric patients? AJR 176:297-301

18. Kamel IR, Hernandez RJ, Martin JE, Set al. (1994) Radiation dose reduction in CT of the pediatric pelvis. Radiology 190:683-687

19. Ambrosino MM, Genieser NB, Roche KJ, et al. (1994) Feasibility of high-resolution, low-dose chest CT in evaluating the pediatric chest. Pediatr Radiol 24:6-10

20. Huda W, Gkanatsios NA (1997) Effective dose and energy imparted in diagnostic radiology. Med Phys 24: 1311-1316 